\title{
$H$ Filter and IMM Algorithm Applied On Target Tracking Problem
}

\author{
Jianfeng Wu, Shucai Huang, Guangjun He and Hongxia Kang \\ Air and Missile Defense College, Air Force Engineering University, Xi'an \\ 710051, P. R. China \\ wjf1331@163.com
}

\begin{abstract}
This paper discusses a new method of HIMM for the target tracking problem. This new method is a combination of the $H_{\infty}$ filter and the interactive multiple model (IMM) algorithm. The sub-filters of IMM algorithm are based on Kalman filter, in which the noise statistical characteristic should been known exactly, and in fact we only can get its approximatively models that worsen the performance of IMM algorithm. However, $H_{\infty}$ filter is not need to carry on any supposition to the noise statistical property, which is not sensitive to the noise, and has robustness to the uncertainty of noise. A meaningful example is presented to illustrate the effectiveness of the authors' method, the performances of IMM and HIMM in terms of stability, tracking accuracy and robustness are compared. The purpose of this paper is to demonstrate the effectiveness of applying the HIMM algorithm on the target tracking problem, which in the past have typically been solved by Kalman filters.
\end{abstract}

Keywords: Target tacking, $H_{\infty}$ filter, interactive multiple model (IMM), Kalman filter

\section{Introduction}

Kalman filter has a very wide range of application in noise signal processing. This filter is established on the basis of $H_{2}$ estimation criterion [1], it requires to know the mechanism of the signal and the statistical properties of the noise, however, can only get the approximate model of the signal in many cases, sometimes unable to obtain the statistical characteristics of the signal, which limit the application of Kalman filter in a certain extent. In addition, Kalman filtering algorithm is the optimal filter for a system without uncertainties, but one of the problems with the Kalman filters is that they may not be robust against modeling uncertainties [2,3].

In the traditional adaptive target tracking algorithms based on single model, although the different model is correspond to the different moving state, but usually only one filter model is in action at each time, model switching fast may bring many questions such as transient error, unidentificate accurately the target state in time etc. Bar-Shalom et al proposed interacting multiple model (IMM) algorithm in 1988 [4-5], the algorithm uses many models of different maneuving characteristic to synthetically descript the moving law of the target, the model transition is described by Markov process, but the sub-filters of IMM algorithm are based on Kalman filter [6]. This filter is on the basis of $\mathrm{H}_{2}$ estimation criterion, which demands to know generation mechanism and statistical characteristics of the noise. However, in many practical cases the approximate model of the signal is only obtained, sometimes the statistical characteristics of the signal are not obtained, which limits the application of IMM algorithm in some extent.

In the presence of modeling uncertainties and external disturbance uncertainties, $H_{\infty}$ filtering can ensure that $H_{\infty}$ norm of the transfer function from noise to estimate error is smaller than a given positive number [7]. Compared with Kalman filtering, $H_{\infty}$ filtering 
does not make any assumptions for spectrum characteristics of jamming signals, it is less sensitive to the model parameters uncertainties and good robustness, and it provides a convenient for the model transition between the different coordinates system.

In this paper, we present a method to deal with linear system filtering with modeling uncertainties and noise uncertainties for target tracking. The main idea is to combine $H_{\infty}$ robust filtering technology with IMM algorithm, namely HIMM.

The paper is organized as follows. In section 2, we describe the $H_{\infty}$ robust filtering algorithm. The general IMM algorithm is reviewed and the calculation steps are given in section 3. In section 4 , we propose a new method to deal with modeling uncertainties and noise uncertainties for target tracking. One experiment is given in section 5 and conclusions are drawn in section 6.

\section{2. $H$ Robust Filtering Algorithm}

Robust control is a control technology which developed for the model uncertainties and external disturbance uncertainties in system. In view of the uncertainty existing in the filtering system, introducing the idea of robust control theory into the process of filtering, lead to the emergence and development of Robust filtering theory, the $H_{\infty}$ filtering is a representative method [8-10].

When the uncertainty of the model and external disturbance exist in the filtering system, $\mathrm{H} \infty$ filtering can ensure $H_{\infty}$ norm of the transfer function between the noises to estimate error is smaller than a given positive number. The spectrum characteristics of the disturbance signal do not make any assumptions, and make the estimation error is minimal in the worst disturbance case. Compared with Kalman filtering, $H_{\infty}$ filter is less sensitive to the uncertainty of model parameters and good robustness. Therefore, $H_{\infty}$ filtering is more suitable than Kalman filter in the process of practical application.

\subsection{Discrete $\boldsymbol{H}$ filtering}

Assume the linear time-varying discrete system is as follows

$$
\begin{aligned}
& x(k)=\Phi(k-1) x(k-1)+\Gamma(k-1) \omega(k-1) \\
& y(k)=H(k) x(k)+v(k) \\
& z(k)=L(k) x(k)
\end{aligned}
$$

where $x(k)$ is the state vector and $y(k)$ is the corresponding observation vector at time $k$, $\Phi(k)$ and $H(k)$ represent the state transition matrix and observation matrix, $\omega(k)$ and $v(k)$ denote process noise and observation noise, $\Gamma(k)$ is the disturbance input matrix. Compared with Kalman filtering, the difference is the third equation, $L(k)$ is the state combination matrix. Because the update process of the filtering covariance relates to the linear combination matrix $L(k)$ in $H_{\infty}$ filter.

Assume that $x(0)$ is the initial state of the system, $\hat{x}(0)$ represents an estimate value of the initial state, the initial estimation error covariance matrix is

$$
P(0)=E\left\{[x(0)-\hat{x}(0)][x(0)-\hat{x}(0)]^{T}\right\}
$$

Here, it is not need to make any assumptions on the statistical properties of $\omega(k)$ and $v(k)$, but both as the unknown disturbance input of the system.

For the filtering system above, the designed filter [11] must satisfies 


$$
\begin{aligned}
\frac{1}{2} \sum_{k=1}^{N}[z(k)-\hat{z}(k)]^{T}[z(k)-\hat{z}(k)] & <\frac{1}{2} \gamma^{2}\left\{\sum_{k=1}^{N}\left[\omega^{T}(k) \omega(k)+v^{T}(k) v(k)\right]\right. \\
& \left.+[\hat{x}(k)-x(k)]^{T} P(0)^{-1}[\hat{x}(k)-x(k)]\right\}
\end{aligned}
$$

where $\hat{z}(k)$ is linear combination of the state filtering $\hat{x}(k), \hat{z}(\mathrm{k})=L(\mathrm{k}) \hat{x}(\mathrm{k})$.

The performance metric of the optimal $H_{\infty}$ filtering is defined as follows

$$
\inf _{\hat{z}} \sup _{\omega, v, x_{0}} \frac{\|z(k)-\hat{z}(k)\|_{2}}{\|x(0)-\hat{x}(0)\|_{2}+\|\omega(k)\|_{2}+\|v(k)\|_{2}}=\gamma_{o p t}
$$

The design thought of suboptimal $H_{\infty}$ filter is: Given $\gamma \geq 0$, firstly it must ensure $\gamma \geq \gamma_{o p t}$, just the problem can have the solution. Through the continuous adjustment of $\gamma$, make it gradually approaching to $\gamma_{o p t}$, which is corresponding to the optimal $H_{\infty}$ filtering estimation problem, so can get the ideal approximate solution of the optimal $H_{\infty}$ filtering estimation problem.

The performance metric of suboptimal $H_{\infty}$ filter is defined as follows

$$
\inf _{\hat{z}} \sup _{\omega, v, x_{0}} \frac{\|z(k)-\hat{z}(k)\|_{2}}{\|x(0)-\hat{x}(0)\|_{2}+\|\omega(k)\|_{2}+\|v(k)\|_{2}}<\gamma
$$

Because the closed-form solution of the optimal $H_{\infty}$ filter can get only under special circumstances, therefore, the optimal $H_{\infty}$ filtering problem generally is instead of seeking its suboptimal solution.

\subsection{Suboptimal $H$ filtering}

For a given noise suppression index $\gamma>0$, if $[\Phi(k) \Gamma(k)]$ is a full rank, if and only if all $k$ is

$$
P(k)^{-1}+H^{T}(k) H(k)-\gamma^{-2} L^{T}(k) L(k)>0
$$

where $P(k)$ satisfies the recursive Riccati equation as follows

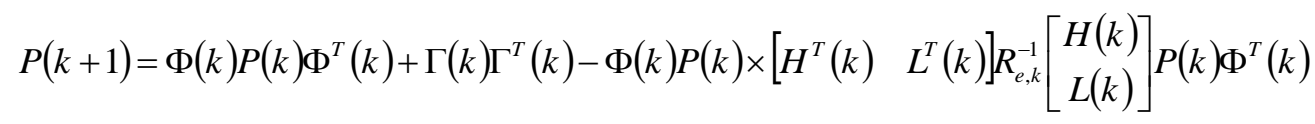

where

$$
R_{e, k}=\left[\begin{array}{cc}
I & 0 \\
0 & -\gamma^{2} I
\end{array}\right]+\left[\begin{array}{c}
H(k) \\
L(k)
\end{array}\right] P(k)\left[\begin{array}{ll}
H^{T}(k) & \left.L^{T}(k)\right]
\end{array}\right.
$$

So the filter is exist and satisfies (5), a possible $H_{\infty}$ filter is

$$
\hat{x}(k+1)=\Phi(k) \hat{x}(k)+K(k+1) \times[y(k+1)-H(k+1) \Phi(k) \hat{x}(k)]
$$

where the filtering gain $K(k)$ is calculated as follows

$$
K(k+1)=P(k+1) H^{T}(k+1) \times\left[I+H(k+1) P(k+1) H^{T}(k+1)\right]^{-1}
$$

\subsection{Comparison of $\boldsymbol{H}$ Filter and Kalman Filter}

Assume that $\omega(k)$ and $v(k)$ are the disturbance input of the system, which are uncorrelated unit white noise process, so the state estimation of Kalman filtering algorithm for the linear discrete time system is 


$$
\hat{x}(k+1)=\Phi(k) \hat{x}(k)+P(k+1) H^{T}(k+1) \times\left[I+H(k+1) P(k+1) H^{T}(k+1)\right]^{-1} \times[y(k+1)-H(k+1) \Phi(k) \hat{x}(k)]
$$

where

$$
P(k+1)=\Phi(k) P(k) \Phi^{T}(k)+\Gamma(k) \Gamma^{T}(k)-\Phi(k) P(k)\left[I+H(k) P(k) H^{T}(k)\right]^{-1} P(k) \Phi^{T}(k)
$$

Comparison of (7) and (12), the following conclusions can be drawn [12]:

(i) The update process of filtering covariance of $H_{\infty}$ filter is related to the linear combination matrix $L(\mathrm{k})$ of the system state. A linear combination of the Kalman filter of arbitrary state estimation is given by a linear combination of the state estimation.

(ii) There is an indefinite covariance matrix $\left[\begin{array}{cc}I & 0 \\ 0 & -\gamma^{2} I\end{array}\right]$ in $H_{\infty}$ filter. Kalman filter can be equivalent considered as adopted unit matrix.

(iii) $H_{\infty}$ filter exists or not is conditional, namely (5) is the filter must be satisfied; due to $L(\mathrm{k})$ will not occur in Kalman filtering problem, and $P(\mathrm{k})$ is positive definite, so the condition is naturally satisfied.

(iv) When $\gamma \rightarrow \infty$, Riccati recursive equation of $H_{\infty}$ filter is simplified to Kalman filtering recursive equation, namely the traditional Kalman filter is a special case of $H_{\infty}$ filter, just $H_{\infty}$ norm becomes very large and poor robustness.

\section{IMM Algorithm}

\subsection{The Principle Of IMM Algorithm}

The basic idea of IMM algorithm [13] is using the different motion models to match different motion states of the target, and using Markov chain to convert various motion models, the global output of the algorithm is the weighted summation of the model probability in the output of each filter. This algorithm effectively overcomes the model error caused by the target motion state does not match the filtering model in the single model tracking. The principle of the algorithm is shown in Figure 1.

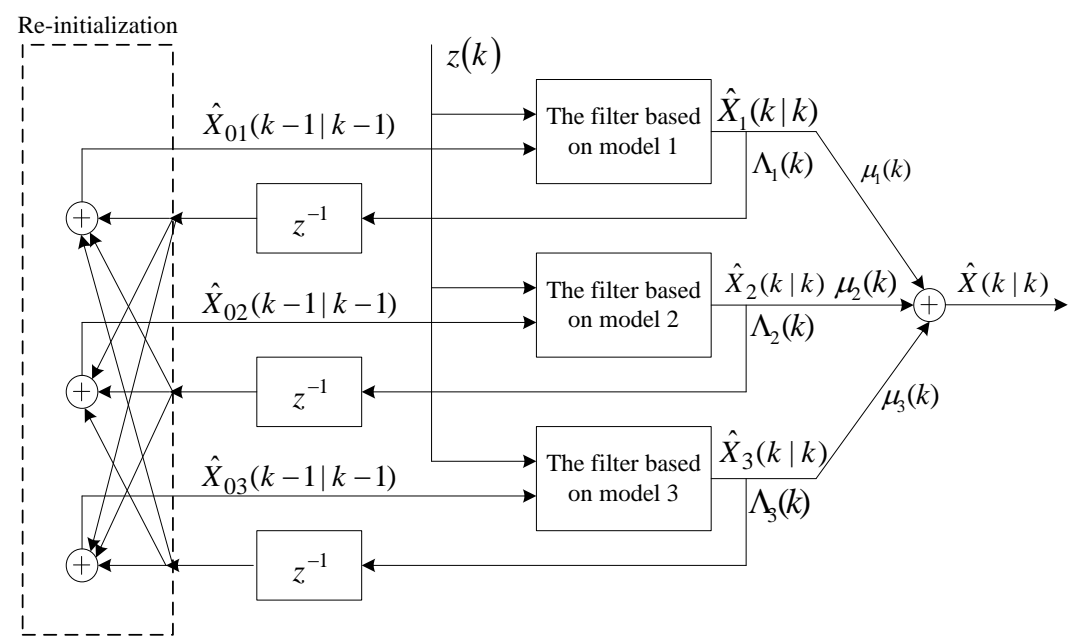

Figure 1. The Principle of IMM Algorithm $(R=3)$

From Figure 1 we can see that IMM algorithm has a strong modular features, in view of different target motion models, you can choose the appropriate target motion model to build the filter, so it has good tracking properties. 


\subsection{The Steps of IMM Algorithm}

Assume that the classical IMM algorithm using $r$ models, so the model $j$ can be described as follows

$$
\begin{gathered}
X(k+1)=\Phi_{j} X(k)+\omega_{j}(k), \quad j=1,2, \cdots, r \\
Z(k)=H_{j} X(k)+v_{j}(k)
\end{gathered}
$$

where $\Phi_{j}$ is the state transition matrix of the model $j, \omega_{j}(k)$ is zero-mean Gaussian white noise with covariance matrix of $Q_{j}$, namely the state noise. $H_{j}$ is the observation matrix of the model $j, v_{j}(k)$ is zero-mean Gaussian white noise with covariance matrix of $R_{j}$, namely the observation noise.

Markov probability transition matrix is

$$
P=\left[\begin{array}{ccc}
p_{11} & \cdots & p_{1 r} \\
\vdots & \ddots & \vdots \\
p_{r 1} & \cdots & p_{r r}
\end{array}\right]
$$

where $p_{i j}$ denotes the transition probability from the model $i$ to model $j$.

IMM estimation algorithm is recursive and each step recursion mainly consists of four steps.

Step 1: the model condition re-initialization

The model condition re-initialization is the assumption that the input of matching filter is mixed determined by the estimates of each filter at prior time under the premise of the $j$ th model is effective at the current time.

(i) Mixed probability

Assume that the matching model is $m_{i}(k-1)$ at time $k-1$, and the matching model is $m_{j}(k)$ at time $k$, the mixed probability is on condition of $Z(k-1)$ calculated as follows

$$
\mu_{i j}(k-1 \mid k-1)=P\left(m_{i}(k-1) \mid m_{j}(k), Z(k-1)\right)=p_{i j} \mu_{i}(k-1) / \bar{c}_{j}
$$

where $\bar{c}_{j}$ is standardized coefficient as follows

$$
\bar{c}_{j}=\sum_{i=1}^{r} p_{i j} \mu_{i}(k-1)
$$

(ii) Mixed estimation

According to mixed estimation [14] the state of re-initialization and covariance matrix is calculated respectively as follows

$$
\begin{gathered}
\hat{X}_{0 j}(k-1 \mid k-1)=\sum_{i=1}^{r} \hat{X}_{i}(k-1 \mid k-1) \mu_{i j}(k-1 \mid k-1) \\
P_{0 j}(k-1 \mid k-1)= \\
\sum_{i=1}^{r} \mu_{i j}(k-1 \mid k-1)\left\{P_{i}(k-1 \mid k-1)+\left[\hat{X}_{i}(k-1 \mid k-1)-\hat{X}_{0 j}(k-1 \mid k-1)\right]\right. \\
\left.\quad \times\left[\hat{X}_{i}(k-1 \mid k-1)-\hat{X}_{0 j}(k-1 \mid k-1)\right]^{T}\right\}
\end{gathered}
$$

Step 2: corresponding to the model $j$, taking $\hat{X}_{0 j}(k-1 \mid k-1), P_{0 j}(k-1 \mid k-1)$ and $Z(k)$ as the input of Kalman filter. Filtering process is as follows 


$$
\begin{aligned}
& \left\{\begin{array}{l}
\hat{X}_{j}(k \mid k-1)=\Phi_{j} \hat{X}_{0 j}(k-1 \mid k-1) \\
P_{j}(k \mid k-1)=\Phi_{j} P_{0 j}(k-1 \mid k-1) \Phi_{j}^{T}+Q_{j} \\
K_{j}(k)=P_{j}(k \mid k-1) H_{j}^{T} S_{j}^{-1}(k) \\
\hat{X}_{j}(k \mid k)=\hat{X}_{j}(k \mid k-1)+K_{j}(k) \gamma_{j}(k) \\
P_{j}(k \mid k)=\left[I-K_{j}(k) H_{j}\right] P_{j}(k \mid k-1)
\end{array}\right. \\
& \gamma_{j}(k)=Z(k)-H_{j} \hat{X}_{j}(k \mid k-1) \\
& S_{j}(k)=H_{j} P_{j}(k \mid k-1) H_{j}^{T}+R_{j}
\end{aligned}
$$

where $\gamma_{j}(k), S_{j}(k)$ is the measurement prediction residual (or innovation) and its covariance matrix respectively.

In the application of the above filtering formulas, it needs to specify the initial filtering conditions, according to the initial state of the target to establish the initial filtering estimation. However, in practice, usually the initial states of the target are unknown, and then the last few observation values can be used to obtain the state initial estimation.

Step 3: the update of model probability

$$
\begin{gathered}
\mu_{j}(k)=\frac{1}{c} \Lambda_{j}(k) \sum_{i=1}^{r} p_{i j} \mu_{i}(k-1)=\Lambda_{j}(k) \bar{c}_{j} / c \\
c=\sum_{j=1}^{r} \Lambda_{j}(k) \bar{c}_{j} \\
\Lambda_{j}(k)=\frac{1}{\sqrt{2 \pi\left|S_{j}(k)\right|}} \exp \left\{-\frac{1}{2} \gamma_{j}^{T}(k) S_{j}^{-1}(k) \gamma_{j}(k)\right\}
\end{gathered}
$$

where $c$ is normalized constant, $\Lambda_{j}(k)$ is the likelihood function matching to $m_{j}(k)$.

Step 4: estimation fusion

The overall estimation and overall estimation error covariance matrix at time $k$ is respectively calculated as follows

$$
\begin{gathered}
\hat{X}(k \mid k)=\sum_{j=1}^{r} \hat{X}_{j}(k \mid k) \mu_{j}(k) \\
P(k \mid k)=\sum_{j=1}^{r} \mu_{j}(k)\left\{P_{j}(k \mid k)+\left[\hat{X}_{j}(k \mid k)-\hat{X}(k \mid k)\right] \times\left[\hat{X}_{j}(k \mid k)-\hat{X}(k \mid k)\right]^{r}\right\}
\end{gathered}
$$

\section{HIMM Algorithm}

As can be seen from the previously mentioned, the sub-filters of IMM algorithm are based on Kalman filtering. So they all have a common characteristic that the state noise and measurement noise covariance matrix is known, both they obey the Gaussian distribution, and these parameters are directly applicate in the filtering process. However, in practice, these assumptions have great man-made factors, when the assumptions are not match to actual noise, filtering performance of the algorithm will be greatly degraded, and may even lead to filtering divergence. For these above reasons, a new method is proposed 
for maneuving target tracking with unknown noise statistics characteristics. The basic idea is to combine $H_{\infty}$ robust filtering technology with IMM algorithm, namely HIMM.

Because the statistical characteristics of the noise is unknown in the filtering process of $H_{\infty}$ filter, so the prediction residual $\gamma_{j}(k)$ is likely to become great at one time, but the error covariance matrix $S_{j}(k)$ is based on the prediction value of the previous time. At this time, there will be a mismatch between $\gamma_{j}(k)$ and $S_{j}(k)$, and resulting in the numerical overflow phenomenon because of the filtering error rapid change in the process of the matching probability calculation. For this reason, the paper studies a numerical robust method to calculate the model probability in reference [15], namely calculating the exponent terms only when calculating the model probability, and uses it in HIMM algorithm.

The model sets of HIMM algorithm are basically same as IMM algorithm, like (13), (14), only the state noise and measurement noise are no longer Gaussian noise in the model sets, but are independent and energy bounded. Similar to IMM algorithm, HIMM algorithm is divided into four steps.

Step 1: the model condition re-initialization

(i) The mixed probability

We assume that the model probability of the model $m_{i}(k-1)$ at time $k-1$ is:

$$
\begin{gathered}
\mu_{i}(k-1)=e^{-a_{i}(k-1)} \\
a_{i}(k-1)=-\ln \mu_{i}(k-1)
\end{gathered}
$$

When the matching model is $m_{j}(k)$ at time $k$, and its mixed probability is calculated as follows:

$$
\left\{\begin{array}{l}
\mu_{i j}(k-1)=p_{i j} e^{-\left[a_{i}(k-1)-A_{j}-\alpha_{j}\right]} \\
A_{j}=\min \left\{a_{i}(k-1): p_{i j} \neq 0\right\} \\
\alpha_{j}=-\ln \left[\sum_{i} p_{i j} e^{-\left[a_{i}(k-1)-A_{j}\right]}\right]
\end{array}\right.
$$

(ii) Mixed estimation

The state of re-initialization and covariance matrix is calculated like (18), (19).

Step 2: corresponding to the model $j$, taking $\hat{X}_{0 j}(k-1), P_{0 j}(k-1)$ and $Z(k)$ as the input of Kalman filter.

Firstly given $\gamma>0$, and judge whether $P_{0 j}(k-1 \mid k-1)$ satisfies (29) as follows:

$$
P_{0 j}(k-1)+H_{j}^{T} H_{j}-\gamma^{-2} L_{j}^{T} L_{j}>0
$$

Due to predict and estimate all the state information of the target, so $L_{j}=I$ in (31). If (31) is not set up, it proves that given $\gamma$ is too small, should increase the value of $\gamma$. If (31) is set up, the filtering covariance matrix can be predicted at next one time as follows:

$$
P_{j}(k)=\Phi_{j} P_{0 j}(k-1) \Phi_{j}^{T}+Q_{j}-\Phi_{j} P_{0 j}(k-1)\left[\begin{array}{cc}
H_{j}^{T} & L_{j}^{T}
\end{array}\right] R_{e, k}^{-1}\left[\begin{array}{c}
H_{j} \\
L_{j}
\end{array}\right] P_{0 j}(k-1) \Phi_{j}^{T}
$$

where 


$$
R_{e, k}=\left[\begin{array}{cc}
R_{j} & 0 \\
0 & -\gamma^{2} I
\end{array}\right]+\left[\begin{array}{c}
H_{j} \\
L_{j}
\end{array}\right] P_{0 j}(k-1)\left[\begin{array}{cc}
H_{j}^{T} & L_{j}^{T}
\end{array}\right]
$$

The estimation of target state based on $H_{\infty}$ filter is:

$$
\hat{X}_{j}(k)=\Phi_{j} \hat{X}_{0 j}(k-1)+K_{j}(k)\left[Z(k)-H_{j} \Phi_{j}(k) \hat{X}_{0 j}(k-1)\right]
$$

where $K_{j}(k)$ is the filtering gain:

$$
K_{j}(k)=P_{j}(k) H_{j}^{T}\left[R_{j}+H_{j} P_{j}(k) H_{j}^{T}\right]^{-1}
$$

Measurement prediction residual and its covariance matrix is calculated as follows respectively

$$
\begin{aligned}
& \gamma_{j}(k)=Z(k)-H_{j} \hat{X}_{j}(k \mid k-1) \\
& S_{j}(k)=H_{j} P_{j}(k \mid k-1) H_{j}^{T}+R_{j}
\end{aligned}
$$

It should be pointed out that $Q_{j}$ and $R_{j}$ used in the filtering process above are different from the state noise covariance and measurement noise covariance in Kalman filtering, they are given weight coefficient according to the target movement forms and actual noise environment. In order to correspond to Kalman filtering, they are written in the above forms.

Step 3: the update of model probability

The update process of the model matching probability is as follows:

$$
\left\{\begin{array}{l}
\beta_{j}=\frac{1}{2}\left(\gamma_{j}\right)^{T}\left(S_{j}\right)^{-1}\left(\gamma_{j}\right)+\frac{1}{2} \ln \left|2 \pi S_{j}\right| \\
m=\arg \min \left(A_{j}+\beta_{j}+\alpha_{j}\right) \\
\mu_{m}(k)=\frac{1}{\sum_{i} \exp \left[\left(A_{m}+\beta_{m}+\alpha_{m}\right)-\left(A_{j}+\beta_{j}+\alpha_{j}\right)\right]} \\
a_{m}(k)=-\ln \mu_{m}(k) \\
a_{j}(k)=A_{j}+\beta_{j}+\alpha_{j}+a_{m}(k)-\left(A_{m}+\beta_{m}+\alpha_{m}\right) \\
\mu_{j}(k)=e^{-a_{j}(k)}
\end{array}\right.
$$

Step 4: estimation fusion

The overall estimation and overall estimation error covariance matrix at time $k$ is respectively calculated like (26-27).

\section{Simulation Results}

Assume that the target motion in two-dimensional plane and the target initial velocity is set to $600 \mathrm{~m} / \mathrm{s}$. The actual moving situation of the target is described as follows: the motion of the target is uniform motion in a straight line in $0 \sim 200 s$, the target acceleration along $x$ axis and $y$-axis is $10 \mathrm{~m} / \mathrm{s}^{2}$ and $-10 \mathrm{~m} / \mathrm{s}^{2}$ respectively in 200 400s. The motion of the target is uniform motion in a straight line in 400 500s, the target acceleration along $x$-axis and $y$ axis is $-10 \mathrm{~m} / \mathrm{s}^{2}$ and $10 \mathrm{~m} / \mathrm{s}^{2}$ respectively in $500 \sim 700 \mathrm{~s}$. The motion of the target is uniform motion in a straight line in 700 900s. Assume that the target position information is only measurable in the target moving process, and actual measurement data is infected by Gaussian white noise with the standard variance of 500. The sampling period used in this simulation is $1 \mathrm{~s}$, the measurement data of the target motion along $x$-axis and $y$-axis is 
independent each other. Since assume the statistic characteristic of measurement noise is not known in this simulation, so given the standard variance of the noise is 800 in IMM algorithm. For each simulation, a total of 100 Monte Carlo runs were generated to obtain the results.

The movement of the target may be modeled by two different modes: constant velocity (CV) model and Singer model. CV model involves a straight flight path with a constant speed and course, and maneuvering which includes turning or climbing and descending [16]. In this case, maneuvering will refer to Singer model, where a turn is made at a constant turn rate and speed. The $\mathrm{CV}$ model used for this target tracking problem is given by (39), (40) [17].

$$
\begin{aligned}
& X(k+1)=\left[\begin{array}{llll}
1 & T & 0 & 0 \\
0 & 1 & 0 & 0 \\
0 & 0 & 1 & T \\
0 & 0 & 0 & 1
\end{array}\right] X(k)+\omega(k) \\
& Z(k+1)=\left[\begin{array}{llll}
1 & 0 & 0 & 0 \\
0 & 0 & 1 & 0
\end{array}\right] X(k)+v(k)
\end{aligned}
$$

The state vector of the target may be defined as follows

$$
X(k)=\left[\begin{array}{llll}
x(k) & \dot{x}(k) & y(k) & \dot{y}(k)
\end{array}\right]^{T}
$$

The first two states refer to the position and the velocity along the $x$-axis, respectively, and the last two states refer to the position and the velocity along the $y$-axis, respectively.

The Singer model used for this target tracking problem is given by (42) [18].

$$
\begin{gathered}
X(k+1)=\left[\begin{array}{cccccc}
1 & T & 0 & 0 & \frac{1}{\alpha^{2}}\left[-1+\alpha T+e^{-\alpha T}\right] & 0 \\
0 & 1 & 0 & 0 & \frac{1}{\alpha}\left[1-e^{-\alpha T}\right] & 0 \\
0 & 0 & 1 & T & 0 & \frac{1}{\alpha^{2}}\left[-1+\alpha T+e^{-\alpha T}\right] \\
0 & 0 & 0 & 0 & 0 & \frac{1}{\alpha}\left[1-e^{-\alpha T}\right] \\
0 & 0 & 0 & 0 & e^{-\alpha T} & 0 \\
0 & 0 & 0 & 0 & 0 & e^{-\alpha T}
\end{array}\right] X(k)+\omega(k) \\
Z(k+1)=\left[\begin{array}{cccccc}
1 & 0 & 0 & 0 & 0 & 0 \\
0 & 0 & 1 & 0 & 0 & 0
\end{array}\right] X(k)+v(k)
\end{gathered}
$$

The state vector of the target may be defined as follows

$$
X(k)=\left[\begin{array}{llllll}
x(k) & \dot{x}(k) & y(k) & \dot{y}(k) & \ddot{x}(k) & \ddot{y}(k)
\end{array}\right]^{T}
$$

The first two states refer to the position and the velocity along the $x$-axis, respectively, and the next two states refer to the position and the velocity along the $y$-axis, respectively, and the last two states refer to the acceleration along the $x$-axis and $y$-axis, respectively.

Equations (39)-(44) are used to generate the true state values of the trajectory and the measurements for this target tracking problem.

The trajectory estimation of the target is shown in Figure 2, which shows that both IMM and HIMM algorithm are able to follow the trajectory fairly well. The acceleration estimation error of $x$-axis and $y$-axis is shown in Figure 3, the position and acceleration 
estimation error of $x$-axis is shown in Figure 4 (a), the position and acceleration estimation error of $y$-axis is shown in Figure 4 (b).

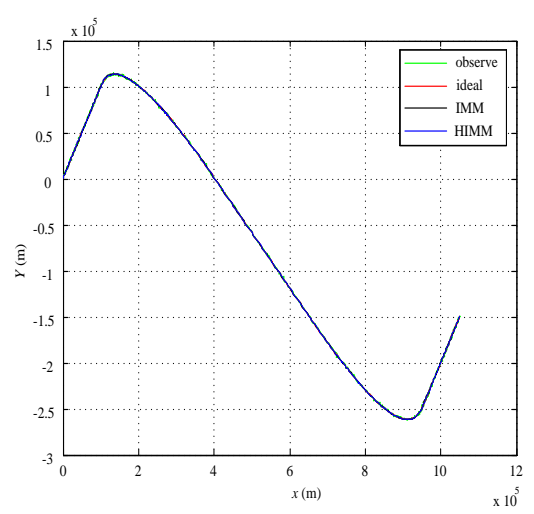

Figure 2. Target Trajectory
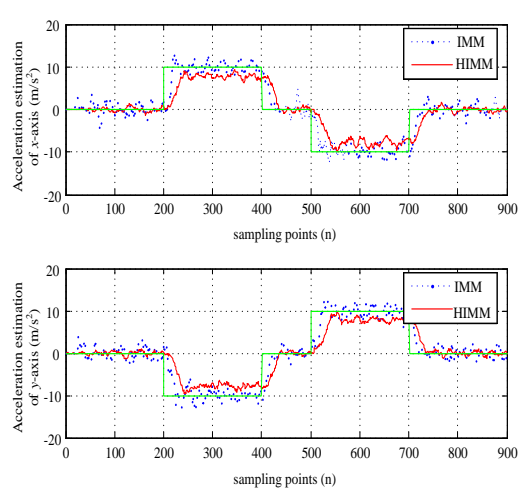

Figure 3. Acceleration Estimation Error
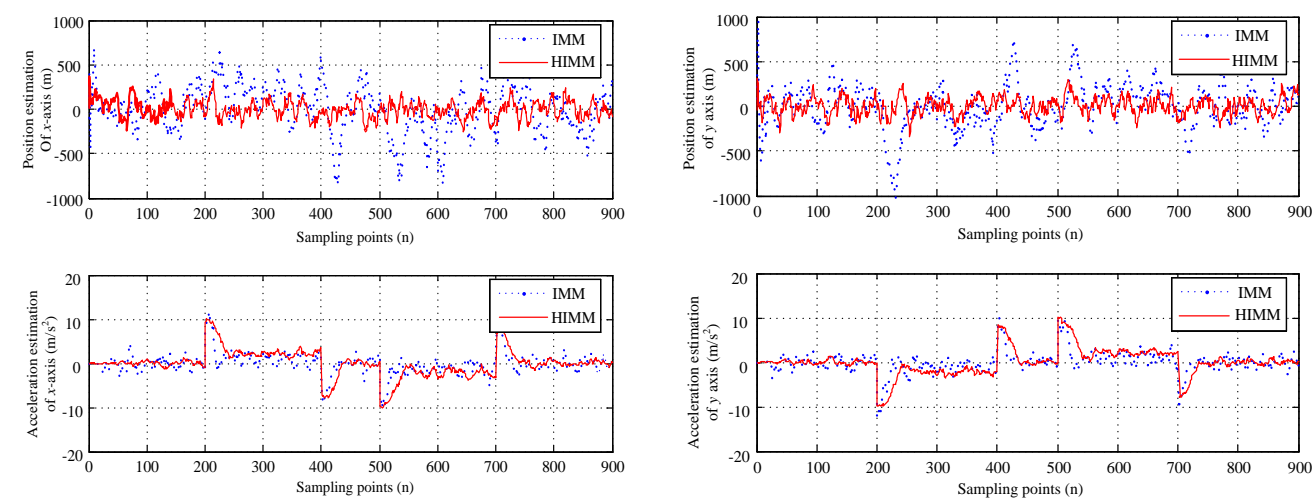

(a) $x$-axis

(b) $y$-axis

Figure 4. Position and Acceleration Estimation Error

From Figure 3-Figure 5, we can see that when the statistical characteristic of the noise is not known, and there is a large difference between a given noise coefficient and actual value, the tracking error of HIMM algorithm based on $H_{\infty}$ filter is lower than that of IMM algorithm based on Kalman filter. Under the effect of interference, the target state estimation of IMM algorithm fluctuates bigger, which shows that the robustness of IMM algorithm is not strong to noise interference. The prediction curve of the target acceleration is relatively smooth in HIMM algorithm, which fully proves that HIMM algorithm is strong robustness to noise interference. The root mean square error (RMSE) results of IMM and HIMM algorithm are shown in Table 1.

Table 1. RMSE of IMM and HIMM algorithm

\begin{tabular}{ccccc}
\hline $\begin{array}{c}\text { Tracking } \\
\text { algorithm }\end{array}$ & $\begin{array}{c}\text { The position } \\
\text { error of } x \text {-axis } \\
(\mathrm{m})\end{array}$ & $\begin{array}{c}\text { The } \\
\text { position error } \\
\text { of } y \text {-axis }(\mathrm{m})\end{array}$ & $\begin{array}{c}\text { The } \\
\text { acceleration } \\
\text { error of } x \text {-axis } \\
\left(\mathrm{m} / \mathrm{s}^{2}\right)\end{array}$ & $\begin{array}{c}\text { The } \\
\text { acceleration } \\
\text { error of } y \text {-axis } \\
\left(\mathrm{m} / \mathrm{s}^{2}\right)\end{array}$ \\
\hline $\begin{array}{c}\text { IMM algorithm } \\
\begin{array}{c}\text { HIMM } \\
\text { algorithm }\end{array}\end{array}$ & 254.9890 & 268.9453 & 2.8627 & 2.9325 \\
\hline
\end{tabular}




\section{Conclusions}

The results of applying HIMM algorithm on the target tracking problem demonstrate its stability and robustness. It is shown that Kalman filtering performs poorly in the presence of modeling uncertainties and noise uncertainties. However, $H_{\infty}$ filtering is able to overcome these difficulties, and provide a stable estimate of the states, so it has a strong robustness and higher tracking accuracy. Likewise, single model appears to be sensitive to model mismatch, but IMM algorithm effectively overcomes the model error caused by model mismatch, the model matching probability is calculated for choosing suitable models to match the target maneuvering. HIMM algorithm combine $H_{\infty}$ robust filtering technology with IMM algorithm, its stability to model mismatch and robustness to modeling uncertainties and noise uncertainties make using HIMM advantageous over the well-known Kalman filter and IMM algorithm. In addition, since using a numerical robust method to calculate the model probability, so the algorithm can effectively prevent the numerical overflow phenomenon appeared in the calculation process. In the past, target tracking problems have typically been solved by Kalman filters. However, the above computer experiment results demonstrate that HIMM algorithm may also be an effective method for handling these types of target tracking problems.

\section{Acknowledgements}

We want to thank the helpful comments and suggestions from the anonymous reviewers. This work was supported by the Nature Scientific fundamental Research Program funded by Shaanxi Provincial Education Department (No. 2012JM8020).

\section{References}

[1] L. H. Xie, "On robust $\mathrm{H}_{2}$ estimation”, Automatica Sinica, vol. 31, no. 1, (2005) March, pp. 1-12.

[2] M. Y. Fu, C. E. de Souza and Z. Q. Luo, "Finite-Horizon robust Kalman filter design", IEEE Transactions on Signal Processing, vol.49, no.9, (2001) January, pp. 2103-2112.

[3] Rodrigo Fontes Souto, Joao Yoshiyuki Ishihara, "Robust Kalman filter for discrete-time systems with correlated noises", Proceedings of the 16th Mediterranean Conference on Control and Automation, (2008) June, pp. 1658-1662, Ajaccio, France.

[4] H. A. P. Blom, Y. Bar-Shalom, "The interacting multiple model algorithm for systems with Markovian switching coefficients", IEEE Transactions on Automatic Control, vol. 33, no. 8, (1988) August, pp. 780783.

[5] Y. Bar-Shalom, K. C. Chang and H. A. P. Blom, "Tracking a maneuvering target using input estimation versus the interacting multiple model algorithm", IEEE Transactions on Aerospace and Electronic Systems, vol. 25, no. 2, (1989) March, pp. 296-300.

[6] E. Mazor, A. Averbuch, Y. Bar-Shalom and J. Dayan, "Interacting multiple model methods in target tracking: a survey", IEEE Transactions on Aerospace and Electronic Systems, vol, 34, no. 1, (1998) January, pp. 103-123.

[7] Z. G. Wu, "Linear robust control theory and calculation", Dalian University of Technology Press, Dalian(2003).

[8] Krishan M. Nagpal, Pramod P. Khargonekar, "Filtering and Smoothing in an $\mathrm{H}_{\infty}$ setting", IEEE Transactions on Automatic Control, vol. 36, no. 2, (1991) February, pp. 152-166.

[9] Xuemin Shen, Li Deng, "Game theory approach to discrete $\mathrm{H}_{\infty}$ filter design", IEEE Transactions on Signal Processing, vol. 45, no. 4, (1997) April, pp. 1092-1095.

[10] Yaesh I, Shacked U, "Game theory approach to optimal linear state estimation and its relation to the minimum $\mathrm{H}_{\infty}$ norm estimation", IEEE Transactions on Automatic control, vol. 37, no. 6, (1992) June, pp. $828 \sim 831$.

[11] L. Yu, "Robust control-linear matrix inequality processing method", Tsinghua University Press, Beijing (2002).

[12] X. P. Wang, X. K. Zhang and L. K. Zhang, "Comparison of $\mathrm{H}_{\infty}$ robust filter and Kalman filter", Journal of Systems Engineering and Electronics, vol. 25, no. 10, (2003) October, pp. 1267-1269.

[13] X. Fu, Y. Jia, J. Du and F. Yu, "New interacting multiple model algorithms for the tracking of the maneuvering target", IET Control Theory and Applications, vol. 4, no. 10, (2010) October, pp. 21842194.

[14] W. D. Blair, G. A. Watson, "Interacting multiple bias model algorithms with application to tracking maneuvering targets", Proceedings of the 31st IEEE Conference on Decision and Control, (1992) 
December, pp. 3790-3795, Tucson, AZ.

[15] X. R. Li, Y. M. Zhang, "Numerically robust implementation of multiple-model algorithms", IEEE Transactions on Aerospace and Electronic Systems, vol. 36, no. 1, (2000) January, pp.266 278.

[16] Bar-Shalom, Y., Rong Li, X., and Kirubarajan, T., "Estimation with applications to tracking and navigation", Wiely, New York (2001).

[17] Lin, X., Kirubarajan, T., Bar-Shalom, Y., and Maskell, S, "Comparison of EKF, Pseudomeasurement Filter and Particle Filter for a Bearings-only Target Tracking Problem", Proceedings of the SPIE Conference of Signal and Data Processing of Small Targets, (2002) August, Orlando, FL.

[18] Singer R. A, "Estimating optimal tracking filter performance for manned maneuvering targets", IEEE Transaction on Aerospace and Electronic Systems, vol. 6, no. 4, (1970) July, pp.473 483.

\section{Authors}

Jianfeng Wu, was born in 1981. He received his B.S. degree in 2003 ,

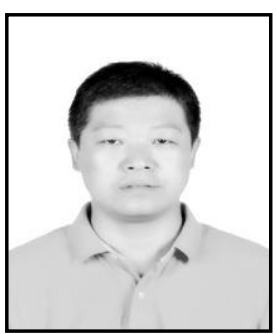
M.S. degree in 2006, from Air and Missile Defense College of Air Force Engineering University. Now he is pursuing his Ph.D. degree from the same university. His main research direction is aerospace coordination detection and intelligent information processing technology.

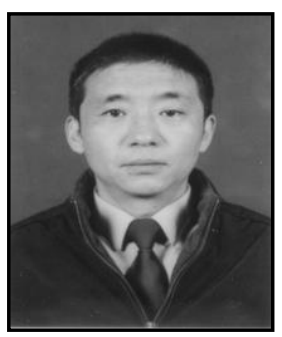

Shucai Huang, was born in 1967. He received his B.S. degree in 1989, M.S. degree in 1992, Ph.D. degree in 2008, from Huazhong University of Science and Technology. Now he is a professor and doctoral supervisor in Air and Missile Defense College of Air Force Engineering University. His research interests include computing signal processing, sensor management and pattern recognition.

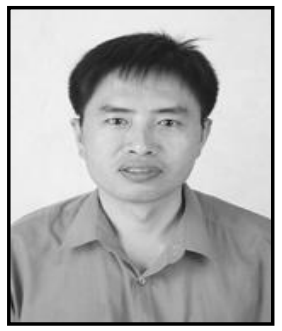

Guangjun He, was born in 1966. He received his B.S. degree in 1988, M.S. degree in 1991, Ph.D. degree in 2008, from Air and Missile Defense College of Air Force Engineering University. Now he is a professor and a doctoral supervisor in Air and Missile Defense College, Air Force Engineering University. His research interests include stochastic signal processing, adaptive filtering and target tracking.

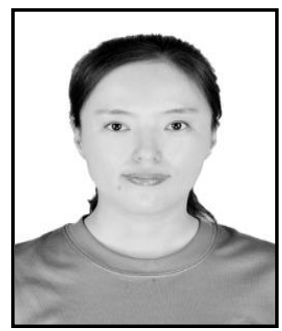

Hongxia Kang, was born in 1980. She received her B.S. degree in 2003, M.S. degree in 2006, from Xi'an Technology University. Now she is pursuing her Ph.D. degree from Air and Missile Defense College of Air Force Engineering University. Her research filed includes signal processing and pattern recognition. 